\title{
Are patient surveys valuable as a service- improvement tool in health services? An overview
}

This article was published in the following Dove Press journal:

Journal of Healthcare Leadership

26 May 2012

Number of times this article has been viewed

\section{Anjali Patwardhan' \\ Charles H Spencer ${ }^{2}$}

Nationwide Children's Hospital Columbus, ${ }^{2} \mathrm{O}$ hio State University, Columbus, $\mathrm{OH}$, USA
Correspondence: Anjali Patwardhan 83I East Mound Street, Columbus, $\mathrm{OH} 43205$, USA

Tel + I 6142662187

Email doctoranjali@hotmail.com

\begin{abstract}
Improving the quality of care in international health services was made a high priority in 1977. The World Health Assembly passed a resolution to greatly improve "Health for all" by the year 2000. Since 1977, the use of patient surveys for quality improvement has become a common practice in the health-care industry. The use of surveys reflects the concept that patient satisfaction is closely linked with that of organizational performance, which is in turn closely linked with organizational culture. This article is a review of the role of patient surveys as a quality-improvement tool in health care. The article explores the characteristics, types, merits, and pitfalls of various patient surveys, as well as the impact of their wide-ranging application in dissimilar scenarios to identify gaps in service provision. It is demonstrated that the conducting of patient surveys and using the results to improve the quality of care are two different processes. The value of patient surveys depends on the interplay between these two processes and several other factors that can influence the final outcome. The article also discusses the business aspect of the patient surveys in detail. Finally, the authors make future recommendations on how the patient survey tool can be best used to improve the quality of care in the health-care sector.
\end{abstract}

Keywords: patient surveys, quality improvement, service gaps

\section{Introduction}

To evaluate different aspects of clinical and nonclinical care, patient surveys have been used extensively. ${ }^{1-8}$ Defining quality improvement from patients' perspectives provides better value for their money with improved safety, accessibility, equity, and comprehensiveness of care. From a provider's point of view, quality improvement may be more efficient, providing more effective services to a greater number of consumers with a reasonable level of satisfaction, enough for customer retention. Patient surveys are the documents produced that reflect the outcome of patient and provider efforts to achieve their independent goals for quality improvement. Providers use these tools to make the necessary changes to make their service more patient friendly. The effectiveness of patient surveys depends on several factors, such as design, standardization, type, construct validity, internal/external validity, reliability, and reproducibility. ${ }^{9}, 10$ The organizational culture and the training status of the surveyors and survey analysts have a significant bearing on the effectiveness of the tool as well as its utility as a quality-improvement tool. ${ }^{11,12}$ This article evaluates the value of patient surveys as a quality-improvement tool in different health-care environments and settings. 


\section{Historical view}

In the United States, the history of surveys dates back to the early 1890s. During that period, medical education and health care for the most part were controlled and managed by for-profit organizations, and quality of care was not considered an essential aspect of the care. ${ }^{13}$ The first report on the poor quality of care of patients was published in 1910 by the American Medical Association. This article was the result of research conducted by researcher Abraham Flexner, who published a report to the Carnegie Foundation. ${ }^{14,15}$ In the same year, Ernest Codman from Boston General Hospital introduced the concept of using patient opinion in improving standards of patient care. In 1917, his efforts led to the development of the Hospital Standardization Program through the American College of Surgeons. ${ }^{16,17}$ In 1952, several North American organizations, associations, and regulatory bodies - the Canadian Medical Association, the American College of Physicians, the American Medical Association, the American Hospital Association, and the American College of Surgeons - joined to form the Joint Commission on Accreditation of Standards and Quality of Care. The main focus of evaluations was based on the patient's perceived view on quality of care.

The concept of improving health-service quality of care in the international arena received attention in 1977, when the World Health Assembly proclaimed the goal of improvement of "Health for all" by 2000. ${ }^{18}$ These objectives were further developed in 1984 for member countries. ${ }^{19}$ One of the objectives was for each country to develop effective policies and procedures that enhanced continuous quality-improvement and quality-assurance mechanisms focusing on availability, accessibility, and quality of care in that country. ${ }^{20}$ The International Society for Quality in Health Care, under the guidance of the World health Organization (WHO), published a report on benchmarking various quality-improvement practices in member states and other countries.

\section{Why are patients' opinions so important?}

There are a number of factors that have made it difficult for the health-care industry to achieve customer satisfaction and retention in the last two decades. These include increasing patient awareness and knowledge, new research and innovations in the health-care field, the increasing cost of services, and continuous competition among health-care providers. Yet continuously improving quality to make services more efficient, effective, and consumer friendly is not an option but a necessity for health-care providers. ${ }^{21,22}$ This disconnect between the continuing quality-improvement imperative and the difficulty the health-care industry has had achieving such improvements has conceded the ultimate power and control into consumer hands, making measurement of customer satisfaction the primary mechanism to drive these needed changes. Customer complaints analysis, satisfaction/ experience surveys, and patient-assessed outcome tools have been made the yardsticks of performance and pointers directing changes. In the evolving relationship between physicians and patients, the terms "consumer" and "provider" seem to be becoming more and more relevant and may represent the most accurate drivers of expectations and boundaries within the relationship. ${ }^{23}$ Weingart et al in 2006 defined service quality as the "patient's self-reported experience of care" as the metric for evaluating quality. ${ }^{24}$ At the same time, the patient is the customer in the health-care industry, but not all customers are patients; several other internal and external stakeholders can also be customers.

\section{Current opinion on the effectiveness of patient surveys}

The Health Evidence Network is an information service of the WHO's Regional Office for Europe and is the decisionmaker for public health and health care in the European Region. ${ }^{24}$ In its commissioned work report titled How Can Hospital Performance Be Measured and Monitored?, the Health Evidence Network states that "The principal methods of measuring hospital performance are regulatory inspection, public satisfaction surveys, third-party assessment, and statistical indicators, most of which have never been tested rigorously. Evidence of their relative effectiveness comes mostly from descriptive studies rather than from controlled trials. The effectiveness of measurement strategies depends on many variables including their purpose, the national culture, how they are applied and how the results are used."25 This statement emphasizes the lack of rigor in this area with uncertainty about the value of customer surveys as qualityimprovement tools and underscores the fact that survey effectiveness may be dependent on several variables, such as how the surveys are applied and how their results are used.

In that publication, it is also noted that "Standardized surveys of patients and relatives can reliably measure hospital performance against explicit standards at a national level. Hospital performance is becoming more focused on health education, patient empowerment, comfort, complaint mechanisms and continuity of care." Yet the report goes on further to comment: "However, traditional satisfaction surveys have 
been methodologically weak, and focused on the agenda of clinicians and managers rather than patients. A review of 195 published studies suggested that few patient surveys were both valid and reliable, and governments may be reluctant to publish adverse results for public hospitals. This agrees with the worries of several researchers on the real cost-effectiveness of using patient surveys. ${ }^{25-29}$ At the same time, other researchers consider patient surveys as ultimate weapons in the improvement of quality of care. ${ }^{30-38}$

It is not clear what and where the gaps are in the understanding of this tool. Why should the researchers and policymakers have such diverse and conflicting opinions about the effectiveness of the patient-survey tool?

\section{Current state}

Most parts of the Western world still use patient surveys to guide their improvement efforts. In 2002, the USA Centers for Medicare and Medicaid Services in association with the Agency for Healthcare Research and Quality pioneered a nationwide, standardized, and publicly reported patient survey to gather patients' experiences of hospital-based care. The 27-point version of this survey was endorsed by the National Quality Forum in 2005. This survey, the Hospital Consumer Assessment of Healthcare Providers and Systems (HCAHPS) survey, in short, "H-caps," aimed to improve quality of care for adult patients. The results of this survey were first reported in 2008. ${ }^{28,39,40}$

HCAHPS surveys were launched with a mission to "empower consumers with quality-of-care information to make more informed decisions about their health care, and encourage providers and clinicians to improve the quality of health care ..." ${ }^{39}$ The surveys are based on provider accountability and patient-experience-driven hospital quality-improvement initiatives. The results of the surveys are reported in the public domain on Centers for Medicare and Medicaid Services' Hospital Compare website. This was believed to generate healthy competition amongst providers to do better every time, and provided patients with choices, options, and opportunities to make informed decisions about their own medical care. This process was implemented after extensive background fieldwork and pilot testing in three states before finally being implemented nationwide in the US.

\section{Disadvantages of HCAHPS patient surveys}

To be comprehensive, the survey has to be rather lengthy, eg, the original HCAHPS surveys had 35 to over 90 questions, depending on the type of survey. This high number of questions may result in poor compliance and response rate. These surveys are carried out either electronically, by telephone using active interactive voice response, or through the postal service. Generally speaking, it is agreed that such remotely conducted surveys per se have poor response rates as compared to face-to-face surveys. Standardized national survey formats do not address local issues and are not customized for a particular issue.

Local hospitals often have to add a few more questions specifically relevant to their services, which makes these surveys even lengthier. In spite of this, most organizations still try to combine their own surveys with that of HCAHPS to reduce their survey costs. ${ }^{39}$ These surveys for the most part are quantitative, and large numbers are required for their outcome and results to be meaningful. Since these surveys are conducted by individual hospitals or their representative commercial agencies, some providers often believe that the surveys are disruptive to services.

The estimated cost of a stand-alone HCAHPS survey is \$10-\$15 per completed survey (\$3000-\$4500 per hospital, assuming 300 completed surveys) for the mail survey. For the phone survey, it is $\$ 16.67-\$ 20$ per completed survey (\$5000-\$6000 per hospital). The active interactive voice response survey appears to cost $\$ 10$ per completed survey (\$3000 per hospital). The average weighted cost per survey may be far higher than this. For example, an individual average weighted cost of a standard mail survey is estimated to range from $\$ 11.00$ to $\$ 15.25$ per completed survey (\$3300-\$4575 per hospital). ${ }^{28}$

This cost can be reduced by combining it with the organization's own survey and reducing the length of standard HCAHPS (27-point) surveys. If this is done, than the validity, reliability, and standardization of the tool will be lost and its responses will no longer remain comparable with those of other stand-alone HCAHPS surveys. ${ }^{28}$ The survey only covers inpatient experience, which is significant but is only a part of total patient care. Also, it is not designed for pediatric age-group patients, who make up a sensitive, vulnerable, and large population group of patients. The responses to the questions can be influenced by the way questions are posed or placed with other questions. If hospitals are allowed to combine the HCAHPS survey with their own survey questions, the responses may lose their standardization.

Abt Associates performed a cost-effectiveness study on the shorter (27-point) version of this type of survey to evaluate its effectiveness and that of public reporting of responses. They specifically looked at the direct impact of public reporting on consumers, the impact of public reporting of 
hospital performance on consumers, and finally the impact of public reporting on hospital quality of care. Apart from the study by Abt Associates, several other well-designed studies have also concluded that there is a significant positive impact from publicizing the results of the patient experience surveys, as well as on the providers' approach, motivation, and performance. ${ }^{26,29,41}$ It was also concluded from these studies that patients want to go for best value (quality, safety, and cost) for their money when they try to purchase health care. Their decisions on which health system to sign up for could be significantly affected by the public reporting of customer/ patient experience with any particular provider. ${ }^{30,42-44}$

Picker Institute Europe is a nonprofit organization that is a prominent contractor for patient surveys at primary and secondary care levels in the National Health Service of England and Scotland..$^{38,45}$ This institute analyzes the results of the surveys and helps organizations make changes in response to customers' feedback. The Picker Institute diligently acquires the information through various sources and activities, such as literature research, focus groups, personal and phone communication with the stakeholders, and looking at the past results of customer reflections and complaints. These activities help them in developing specific questionnaires with excellent face validity, a high degree of construct validity, and a high degree of internal reliability for specific situations. ${ }^{46-48}$

In 2011, UK authorities decided that general practice surveys would be conducted twice a year, but most secondary and tertiary care system surveys would be conducted annually. The results of the surveys are compared from previous years and with other similar provider organizations to explore areas for improvement. The results are published on the website of the provider organization and also on the website of the Care Quality Commission. Patients are then able to make informed choices and decisions in choosing the health-care provider that best meets their needs. These Picker surveys also come with all the advantages and disadvantages of quantitative surveys, such as the HCAHPS survey, except that the Picket Institute occasionally conducts face-to-face, quantitative surveys of patients if the research question requires it. Participation in regular patient experience surveys is voluntary in the USA but mandatory in the UK and is linked to UK government financial allocations and funding for hospital systems.

In the UK before 2009, quality of care was monitored and regulated by the Healthcare Commission, which was established on April 1, 2004. The Healthcare Commission was a replacement for the Commission for Health Improvement, which existed from 2000 to 2004. In 2009, the UK government established another public body called the Care Quality Commission, which was designed to regulate health- and social-care services in England and was a replacement for both the Healthcare Commission and the Commission for Social Care Inspection. Patient surveys are used extensively by various National Health Service organizations and general practice surgeries to evaluate their services and identify areas for improvement. As mentioned, it is now common practice in the UK to publicize the results of patient surveys. The Care Quality Commission reviews the survey results on a regular basis and makes suggestions for necessary actions to improve care.

\section{Recent UK surveys}

Overall, the system for collecting customer/patient feedback on their experiences with providers and using that to improve quality of care and make the care more customer friendly is more structured, uniform, and robust in the UK than in the USA at this time. Weingart et al in the UK used a quantitative face-to-face survey technique on 228 adult inpatient admissions. ${ }^{24}$ Written consent was obtained from each patient. The patients were visited by the surveyor two to three times per week for 5 minutes during the hospital stay and were contacted by telephone 10 days after discharge. Four questions were asked of the respondents during these visits:

1. Did you experience any problems with your care during this hospitalization?

2. Were you hurt or stayed in the hospital longer than necessary because of problems with your care?

3. Did any error by the provider affect your quality of care adversely?

4. Rate your overall quality of care during hospitalization on a 5 -item scale (from poor to excellent).

In this study, 228 patients were interviewed, and these interviews generated a total of 912 reports. Out of these 912 reports, 157 reports were classified as expressing concerns or dissatisfaction, with $38.6 \%$ of the 228 patients experiencing at least one dissatisfying incident (range 1-6) and $40(17.5 \%)$ experiencing two or more.

This study was innovative, but there were many problems:

1. The survey methodology was quite labor-intensive.

2. Most of the questions asked were closed-ended questions, and there was limited opportunity for survey takers to communicate and express their feelings, with only a comment section allowing some free text at the end of the survey. Thereby, the survey negated some of the advantages of the face-to-face interview. 
3. The data were collected in a semiqualitative way, based on raw data and percentages without using any analytic statistical method. Problems with the data need to be assessed by using either conventional statistics or statistical process control charts.

4. There were no controls from past or other similar groups to compare performance.

5. Even if concerns were recognized (such as poor communication, environmental issues, food complaints, unclean and poor sanitary conditions, poor interpersonal skills, unprofessional behavior), it was impossible to incorporate these concerns into a quality-improvement plan in the absence of details of the incidents.

6. The survey methodology was not standardized, nor were the validity, effectiveness, reliability, and reproducibility responsiveness of the questionnaire evaluated.

7. The questionnaire was too short, was not comprehensive, and did not cover all relevant areas of care. Patient dissatisfaction alone does not give enough opportunities to explore what actions can be taken so that similar customer dissatisfaction incidents can be prevented.

To address the specific needs of a particular service, several customized questionnaires have been developed by researchers. ${ }^{31,33-36,49}$ Few of these are well-designed studies with good external and internal validity. Dancet and colleagues ${ }^{31}$ in Belgium developed a questionnaire designed for patients with a diagnosis of endometriosis. The 10-dimensional patient-centered endometriosis-care questionnaire was designed after an extensive literature review by the researchers and focus group discussions with subject experts and patients. Demographic questions and free text space were also added to the questionnaire. This online survey questionnaire was piloted in the Netherlands, Italy, and the UK. Various qualitative internal-reliability assessments, such as item analysis, interitem analysis, confirmatory, exploratory factor analysis (EFA), and reliability analysis, were performed before final implementation.

A total of 541 patients participated in the research survey, adding good statistical power to the study. Appropriate statistical methods were applied to the results. The researchers concluded that the 10-dimensional patient-centered endometriosis-care questionnaire might serve as a tool to benchmark patientcenteredness, help to standardize research in cross-cultural European research, and identify areas for improvement. Unfortunately, the authors did not comment on whether this tool was used to improve services, and if yes, then how. ${ }^{31}$

In this study, by including various cultures, external validity was improved. Yet the study was mainly focused on the European population and might not be applicable to African and Asian populations. The article did not include information on the baseline status of the quality of care and also did not report whether the use of the questionnaire resulted in significantly improved quality of patient care. Thus, the questionnaire was valid and reliable for identifying the status of the quality of patient-centered care, but its efficacy as a quality-improvement tool was established in the absence of information on prospective validation of the tool. ${ }^{31}$

Consumer Assessment of Healthcare Providers and Systems (CAHPS) surveys are used widely in the USA for collecting a patient's perspective of their ambulatory (including pediatric ambulatory) and hospital care experience. They were designed in 1995 by the Agency for Health Care Policy and Research with the goal of improving the quality of care and to make it more patient focused by using a patient perspective on the care each received. ${ }^{34}$ It was suggested by several subject-matter experts that this tool might not be valid for developmental and preventive pediatric health care.

There are fewer tools validated or even available for the US pediatric population, which has its own challenges. ${ }^{37}$ The cognitive challenges in understanding children's needs, language barriers in expression of children's thoughts, their often-short attention span, an obvious sensitivity and vulnerability to harm, and inability to decide for themselves in their best interest does place them in a separate bracket of consumers. The specific article cited here is about development and testing of a CAHPS survey applicable to the developmental and pediatric preventive health-care area. ${ }^{37}$ The subject-matter experts developed the pediatric tool after conducting two focus groups and nine cognitive interviews. A dual-language field test of the instrument was performed with 670 parents before being implemented. The tool was found to have reasonable internal consistency (coefficient $\alpha$ ) and interphysician reliability. It has been accepted nationally as a measure of quality of pediatric care.

\section{Survey weaknesses}

There are no baseline data yet on the status of quality of care and how this instrument (CAHPS Survey) has been used to improve the quality of care in the pediatric population. Similarly, most published US surveys on the customer/patient experience end at the point where they suggest how they can identify gaps in the quality of care, but do not go further to delineate how they measured improvements resulting from the use of this survey tool and how these identified gaps might be further closed. Also, after the patient experience survey tools have been used in practice, there has been little effort 
to evaluate the interaction between those tools and the work environment, which may affect the tools' reliability/validity/ reproducibility in the real world. ${ }^{50}$ If these tools are evaluated better, opportunities for further refinements may be identified. But this is only possible when the data describe the actual effect and outcome of these surveys on the quality of care and the comparison of new data to baseline data or the previous year's results.

A patient's perception of their experience with the provider is all about the provider's ability to manage the expectations of the patient. Patient and provider psychology is integral to the process. Unfortunately, most survey tools have been developed without consideration of psychological issues and input from psychologists when developing an instrument. ${ }^{47,51,52}$

Several customer/patient surveys have been performed that have identified gaps in service provision without specifying follow-up changes to address these problems with the quality of care. ${ }^{53}$ At other times, it appears the customer satisfaction/patient satisfaction surveys were just used to affirm if the services offered by the provider were satisfactory. The providers also appear to use these survey results only for marketing purposes. These are mostly online, email, or telephone surveys done soon after the services are delivered. The response rate is sometimes very poor, and responses are recorded to closed-ended questions as "satisfied" or "not satisfied" for the long list of services that may have been provided to the patient during her/his time with the provider. ${ }^{49}$ The results are often publicized as a high number of patients strongly agreeing that the care provided was up to their satisfaction. This use of surveys actually serves no purpose other than financial and can sometimes be deceiving. This type of survey outcome should never be used as a quality-improvement tool. These surveys avoid the important questions. What matters is not how many patients are really satisfied with the services of the provider, but rather those remaining dissatisfied patients and any unanswered questions about why they are dissatisfied and how services could be improved.

On the other hand, an association has been identified between dissatisfaction levels and patient characteristics. But this association does not provide a detailed evaluation of patient responses in the correct context and its specific background, as their dissatisfaction might not reflect the real gaps in the quality of care. ${ }^{50,54,55}$ Several researchers have proved that some patient characteristics, such as age, reported health status, gender, education level, and ethnic background, all affect real or perceived dissatisfaction in their experience with the provider. In some studies, these factors accounted for as much as $3 \%-8 \%$ of the variation in results in an average population, but this effect could be larger if the variation in the population is large. ${ }^{54-57}$

Analysis of many survey tools for quality improvement and how they are used often reveals that surveys are full of biases and designing errors. These errors lead to collection of haphazard, unintended information, leading to an inability to bring about desired outcomes.

Designing the questionnaires, phrasing of the questions, sequencing, length, method of implementation, and psychometric properties of the questionnaires - can all influence the responses. The skills and training level of the person who develops the questionnaire as well as input from subject experts and psychologists can affect the quality and effectiveness of the survey. ${ }^{10,58}$

\section{Selection and nonresponse bias}

These surveys are constructed to explore specific questions regarding service delivery, which, for example, might be satisfaction with those particular services or agreeing with any recent changes. They are constructed for a specific sample of patients, eg, 80\% Caucasian, 10\% black, 5\% Hispanic, and $5 \%$ Asian. Another population might have 58\% females and $42 \%$ males, as indicated in the structure of local community demographics. This technique is called desired representative population.

Poor survey population sampling may result in undercoverage (having fewer target patient participants). Voluntary response bias occurs when taking the survey is voluntary. Patients who have strong opinions will be overrepresented, skewing the outcomes. ${ }^{59}$ Convenience sampling is a phenomenon whereby people who are easily approachable are surveyed, such as patients on a common mailing list or all patients in an outpatient setting. As these groups may not represent the target representative population, the survey results cannot be used to improve the quality of care for the target population. Another example might be surveying patients in a gynecology outpatient clinic in order to improve the services of an orthopedic inpatient surgery service.

It has been observed in general that Caucasian populations are more often satisfied with their health-care services than non-Caucasian populations in several countries. ${ }^{60,61}$ This finding may reflect differences in provider-patient interactions or well-known racial health-care disparity in those societies. ${ }^{53,62-68}$ It has also been shown that older and healthier patients give more positive scores to their providers than 
their counterparts.$^{69}$ It has been observed that patients who live in relatively affluent areas often give lower ratings to their health-care services than patients from middle- and lower-class socioeconomic areas. Also, satisfaction level with the health-care services seems to vary with the level of education of the patient, with the less educated being more satisfied and the more educated being less satisfied with the services provided to them. ${ }^{70}$ Thus, it can be difficult to genuinely evaluate medical services and plan improvement of health-care services using patient surveys. The services may actually be more than adequate, but the expectations of various levels of patients may bias the results.

The external validity and generalizability of these surveys may at times be questionable and the results may be affected if used in a population sample (nonrepresentative sample) that the survey was not designed for. The random selection of responders can improve the quality of a representative sample of participants. This improvement may be limited by the response to the survey. Hence, the responding population may be a nonrepresentative sample introducing a sampling error in the results. ${ }^{71-72}$ Some authors recommend using the Heckman correction, a statistical method developed by James Heckman in 1976 to correct for selection bias in nonrandomly surveyed populations. ${ }^{73}$ The response bias can make the results exceedingly positive if the number of unsatisfied patients is high. This observation is based on the evidence that most unsatisfied patients try not to participate in constructive and quality-improvement activities and responders will be only the patients who are satisfied with the services. This skews the results on the positive side. ${ }^{71}$ This will create an absurdity wherein services or providers who have fewer satisfied patients will paradoxically have a higher satisfaction rate than those who have more satisfied patients. ${ }^{74}$ It is important to remember that nonrespondents are a heterogeneous group, and the frequency of various reasons for nonresponse can influence the vigor and trend of the nonresponse bias. ${ }^{72}$

\section{Effect of type and method of implementation of survey tool}

The length of the survey can have an effect on the response rate, discriminatory power of the survey, reliability (internal consistency, test-retest, and interrater), and construct validity of the instrument. ${ }^{45}$ Although shorter surveys may have a better response rate, they may often have decreased discriminatory power, reliability, and internal consistency. Thus it is very important to design surveys with appropriate length so that they can be used effectively. ${ }^{47,75}$
If questions are constructed with many platitudes, then participants often tend to respond with agreement. This also happens when the subject is such that the response may not be in line with social norms or if they are controversial, eg, questions on abortions. Survey takers may try to give socially acceptable answers without reflecting their own opinion. This is called acquiescence bias or social desirability bias. As a result, positive or socially accepted responses get overrepresented. Double-barreled questions are those that raise more than one issue and respondents are only allowed one answer. This makes evaluation of these questions difficult.

If surveys are conducted in a skewed population area, their results will not be comparable to other geographical areas that do not have a skewed population distribution, eg, in terms of demography or ethnic distribution due to geographical bias. The population selection may be influenced by the type of survey instrument utilized. For example, if telephone surveys are conducted on cell phones, then this may unwittingly underrepresent the elderly population, or if a survey is conducted in the evening, then the outpatient clinic population not open at that time will not be able to participate (temporal bias).

Closed-ended questions sometimes make participants feel compelled to answer the question with a yes or no, in spite of not completely agreeing with the available answers.

Many times, surveys include several counterfeit questions, such as, "Your satisfaction with the services is what percentage?" So even if a patient responds by saying $60 \%$ or $70 \%$, the real meaning of the response is usually unclear. How can providers then utilize this information in a quality-improvement plan? Occasionally, whether to hide problem areas or increase survey completion, but mostly inadvertently, surveys include happy, leading questions. These lead to positive responses.

Response rates vary with the type of the instrument, with the best response rates occurring in face-to-face interview surveys. ${ }^{52}$ Various types of survey tools are designed to be used in appropriately selected diverse situations. Correct selection of the instrument is also vital. The validity of the instrument being used can be increased by using an analysis method called triangulation. ${ }^{76,77}$

\section{Response rates and timing of responses to instrument}

The response rate varies with several factors, such as the length of the questionnaire, the type of the instrument, target audience, and the specific research question. 
Face-to-face and telephone interviews elicit responses immediately, while in email surveys most responses come on the day they are received by the participants, followed by within a week of receiving the survey. It has been observed that surveys sent out in the early morning ( 6 am to $9 \mathrm{am}$ ) get the best response. Response time is shorter if the surveys are fired out in the evening, but response rates are low. Monday mornings and days following national holidays are not the best days to send out online surveys. ${ }^{78}$ If surveys are sent out to a larger population ( $>1000$ people), the personal touch may be lost. When surveys are targeted at large groups, there are risks that patients may not feel their areas of interests are covered in the questionnaire. They may find the survey too vague and not worth spending the time to complete.

\section{Barriers to surveys}

Various process barriers have been identified that can affect the outcome of a survey. These barriers include language, educational and comprehension level, and comorbidities such as cognitive deficiencies, psychiatric problems, or central nervous system diseases. There are physical deficiencies that might impact the writing and reading capacity of the participant. Alcohol or drug abuse may influence the response results, as well as nonresponder rate, of the participants. $^{79}$

There are other barriers. Researchers have observed differences in the response rate in different demographic populations. Health-care providers and health-care provider organizations are often threatened by public comparison of their patient survey data. The survey results are often not comparable because of the heterogeneous nature of the populations and communities and the type of survey tools used to evaluate the satisfaction levels of the patients. It cannot be overemphasized that valid comparisons of various hospitals' performance can only be made when adjustments for patient mix, services mix, and the type of survey are made. ${ }^{40,80}$

\section{Rigging}

In certain health-care systems, providers are compensated or incentivized based on the patient satisfaction survey results. ${ }^{81}$ These providers occasionally have been observed to manipulate the design, analysis, and results of survey results to improve outcomes and obtain higher scores. ${ }^{82}$ Other organizations use their patient satisfaction surveys for competitive marketing and publicity. Sometimes, the fear of any negative publicity and the need to publicly display their results may push providers to introduce bias in questions, ie, a design bias of the questionnaire. Thus, questions may be structured in a way to elicit a specific response that sways the outcome of the survey towards a positive or desired side for the provider, thereby destroying the survey's validity. This approach will not necessarily help in improving the system, nor will it improve real satisfaction in the patients. ${ }^{83}$

There are so many other factors that may alter the real results of a survey. These include fear of demotion or loss of job, criticism for poor performance, performance-linked incentives and bonuses, competition between providers, compulsion to publish the survey results, or even just lack of an outside perspective. These survey efforts often result in either collection of infective information (results fail to identify real gaps in the service provision) or un-usable information (difficult to translate the results in to an quality improvement project which can bring desired improvement in the quality of care). On the contrary sometimes the results may mislead the quality improvement efforts and resources in the wrong direction. ${ }^{28}$

\section{Disconnect between survey outcomes and implementation of results}

The scenario raises two questions. The first is "if patient experience surveys can identify the gaps in the service provision and secondly can the results of patients experience surveys be used to improve the quality of care in the healthcare setting". A second way these questions and concerns could be interpreted is "if patient experience surveys have been used to identify the gaps in the service provision and secondly if the results of patients experience surveys have been used to improve the quality of care in the healthcare setting"

The first interpretation of the research question is hypothetical, and the answer is affirmative in the ideal world. Patient experience surveys can be used to identify gaps in service if constructed in the right way (right kind of survey tool), applied in the right environment, and with the right consumer group. Surveys must be used at the right time, in the right settings, and evaluated in the right way.

The response to the second part of the question is again affirmative in the ideal world; patient experience survey results can be used as quality-improvement tools and thereby help improve service provision in health-care settings. But, and this is an important but, the results of these surveys must be used in follow-up quality-improvement projects using relevant quality-improvement methodologies and tools. 


\section{Surveys and the real world}

However, in the real world this does not always happen. Patient experience surveys are used extensively and regularly for almost every specialty and field of health care. Some of these surveys are well constructed, standardized, and validated to be able to compare the performance of various health-care organizations and make the area competitive. ${ }^{38,39}$ Some organizations have their own customized surveys addressing their local and individual needs. But other organizations add their own customized questions to standardized and validated national surveys to reduce the cost of the surveys and to improve the response rate. This technique is not in the long run a good way to reduce cost because it renders an organization's survey results noncomparable with other organizations, and the inter-question interaction and sequencing can alter the responses and response rate. Whichever way it is done, it appears clear that most surveys are reasonably able to identify gaps in service provision, but not all results are translated into quality-improvement projects that have a good chance of improving medical care.

Survey results may have both positive and negative outcomes. There are several published reports of these results suggesting how effective a survey was in identifying areas for improvement. Unfortunately, the published literature lacks evidence of how these survey results were used for change through various quality-improvement projects and how the continuous quality-improvement process was monitored and horizontally deployed in other geographic areas and medical institutions.

\section{Improvement in quality of care}

Quality of care is defined in relation to patient expectations and perceptions. Good quality of care is that which meets the expectations of the consumer/patient and leaves him/her satisfied after the service is provided, and hopefully has a relationship to good outcomes. Therefore, customer/patient satisfaction surveys are used to measure the performance of providers as well as patient-centered quality of care. But improvement in quality of care is a different case scenario where a gap in medical care is identified or the effort is made to improve the current quality of care as a part of continuous quality-management agenda.

The first step in the process is to identify the goals and the targets, and next to identify the key performance indicators (KPIs) by which the change can be measured. These KPIs will also inform if the change is an improvement or deterioration from past performance. The final step before implementing change will be to identify what changes are needed in the process/system that will lead to improvement and help to achieve the goals/targets.

After deciding what changes will lead to improvement, changes are sequentially tested on a small scale using several "plan-do-study-act" cycles before the change is implemented. The whole process is then audited to see if the target is achieved and if the desired improvement from the current status has been accomplished. After targets are achieved, the new improved status becomes the current position and a higher target/goal is set for more improvement for the next cycle. This is how continuous quality management/improvement is carried out. In this case, after using the patient experience surveys, the conclusion should not read something like "better response in this field and not so good in other fields"; rather, measures/interventions need to be identified and used to address gaps in service. These measures should then be publicized to indicate how improvement was made rather than publicizing the response rate. ${ }^{84}$

The relationship between a medical-care provider's ability to use patient experience survey tools effectively to identify gaps in service and the ability to use the results of this survey to improve the quality of care in a consumer's eye seems to be complex and nonlinear. Both processes require an understanding of provider organizations and the agreed partnership and relationship with their employees on one hand and patients on the other hand.

The common denominator for success of both parts of the survey tool is that providers and patients must be willing to work as partners in using the survey tool effectively and to make improvements based on the results. Unfortunately, for the most part, the two fail to work in a partnership, frequently because of their own undeclared agendas, conflicts of interest, and several organizational-culture barriers. It is clear from the current published evidence that in spite of serious efforts, few organizations can have genuine partnership with their consumers due to organizational cultural attitudes and even arrogance. ${ }^{85}$ There exists some ongoing frustration amongst the providers as well as patient groups regarding the way surveys have been constructed and conducted, and how the survey results have been used.

\section{Organizational culture and surveys}

Most stakeholders appear to believe that organizational culture has a great impact on the way any survey tool is utilized to collect information, which information is collected, and the way results are used. Organizational culture can be defined in 
several ways, for example: "Organizational culture is the term used to describe the shared beliefs, perceptions, and expectations of individuals in organizations. Because of its shared nature and implicit understanding about organizational norms and values, culture can have a dramatic effect on efforts to change specific procedures or processes." ${ }^{\prime 86}$

Maull et al ${ }^{87}$ defined culture with reference to a number of concepts: as a learned entity, as a belief system, as strategy, and as mental programming. There are several reports in the literature that support the assertion that organizational culture is a very important factor in driving quality-improvement efforts and managing any change. ${ }^{88-91}$

Total quality management is considered an approach for an improved organizational culture and organizational strategic commitment to take active measures to make continuous improvements in service provision and to meet the expectations and needs of current and future customers..$^{92-94}$ Partnership between the provider and patients is an essential ingredient in seeking and achieving this improvement. It has to be a collaborative approach in which both work for mutual benefit by sharing the risks as well as the benefits resulting from the partnership..$^{95,96}$

Investment in training and development of the relevant provider staff in the quality tools and methodologies can have double benefits. This can be seen as an indicator of an organization's policy of greater employee involvement, commitment, and development, and at the same time the correct tools can be utilized for the specific situation to collect desired information and bring about desired change. ${ }^{97}$ Several research studies in sociology and quality in health care have suggested that employee training and development, generally and specifically, in quality measures and tools leads to improved employee commitment, teamwork, and the successful management of quality culture. ${ }^{98,99}$ Research has shown that appropriate training in constructing, selecting, and conducting a survey as well as analyzing survey results greatly increases a survey's effectiveness as a quality-improvement tool. ${ }^{100}$

Similar observations can be found in the literature on faceto-face interview results. The provider group felt that training in survey tools could have improved their performance. Most of the providers were not sure which type of survey should be used in which situations.

The question of whether and how to share results of surveys with the public is interesting. The WHO's Health Evidence Network suggested that this policy showed an intention to empower patients and a commitment to transparency - both laudable goals. These reports attract a great deal of attention from customers/patients and the media, but do not necessarily result in effects on performance improvement of the organization or provider. This sharing of survey results also does not appear to have any effect on the behavior of patients or providers, or on performance outcome. ${ }^{27}$ Sometimes this data, instead of generating provider/patient interest and motivation, may in fact adversely affect referral patterns; the data may also generate defensiveness and trepidation among providers, perhaps affecting their performance adversely. Thus, the publicizing of the results of the surveys has not been found to be particularly useful, yet is still very popular in real life. ${ }^{101,102}$

Trying to get answers for the same question through more than one source improves the reliability of the information. Using more than one type of survey to study a single situation or question can improve the quality of the collected data. This process is called triangulation. ${ }^{103}$

In the UK, all health-care providers are essentially required to commission patient experience surveys. There are national KPIs, and every provider and provider organization is evaluated on these specific KPIs to identify patient-centered care at least once a year. These KPI ratings are used to compare various health organizations and then passed on to the Department of Health. The results are available on each organizational website as well as on the Department of Health website for public review. ${ }^{45,48,104-112}$

\section{Conclusion}

It should be recognized that conducting patient experience surveys and using the results to improve the quality of care are two different processes. The utility of patient surveys as a quality-improvement tool depends upon the successful completion of both these processes.

The effectiveness of the survey instrument depends on appropriate design and application of the right tool in the right situation. The survey response rate and appropriateness of the response are dependent on several factors, such as design (length, standardization, validation, reliability, responsiveness, discriminatory power, and structure of questions) and the characteristics of the desired representative population. Customized, standardized, and validated surveys can be used in the health-care setting successfully as quality-improvement tools. It is not a "one size fits all" type of instrument.

Patient satisfaction surveys can be a waste of time and resources for both patients and provider, because the results often cannot be translated into a quality-improvement plan. 
On the other hand, patient experience surveys can be valueadding and empower patients.

Patient surveys have been incorrectly used to falsely publicize positive results by some organizations and used by some providers to achieve financial gains. This is wasteful, unethical, and a lost opportunity for improvement. The organizational culture and the training status of the surveyors and survey analysts have a huge bearing on a survey's effectiveness as a quality-improvement tool.

It is important for provider organizations to develop partnerships with their employees and with patients, with the aim of building trust and inculcating an attitude of working towards mutual benefit. Transparency and sharing results with all stakeholders helps in persuading and negotiating when required. The measurement system and yardsticks should be consistent, defined, and used to compare all health-care provider organizations nationally and possibly internationally.

Success stories should be shared in relevant conferences, meetings, and peer-reviewed journals. Collaboration and benchmarking should be encouraged to disseminate best practices so that good practices are incentivized nationally and recognized.

Appropriate types of surveys can be used for different situations, depending on the information that a survey is seeking. A single source of information should not be used in isolation to make change or policy decisions; multiple sources of information should be considered to get better results through triangulation. Nonstandardized, nonvalidated, and poor-quality surveys lack reliability, reproducibility, and validity, and may not provide a true picture of the performance of the provider. The results of these surveys may only increase provider anxiety, prompt poor decision-making, and trigger waste of resources when using the survey results to improve the system.

Healthy comparison of the performance of various providers and provider organizations is good practice. The survey aim should still be to manage the performance of the organization and quality of care and not just to use the results for marketing purposes and generating of unreliable rankings and comparisons. The development and use of rigorous standardization of measurement criteria for national and international comparisons is crucial to valid comparisons of provider organizations or institutions. Publicizing surveys has shown little effect on performance improvement of provider organizations or improvement in the return of customers and patient satisfaction.

\section{Future policy implications}

From the viewpoint of policy-making, several recommendations can be made:

- There should be internationally accepted, clear, published, and accessible measures and guidelines for patient experience surveys.

- The practice of surveys in any facility should be audited at regular intervals.

- Strict rules should be formulated nationally or internationally to survey the provider organizations on their customer-centric policies at regular intervals.

- There should be guidelines on how to involve patients in the decision-making and quality-improvement activities of organizations and how to empower them and make them partners in their own care.

- Safeguards and incentives need to be utilized if the provider organizations fail to follow the guidelines.

- Prospective research efforts with better control of confounding factors that are found to be influencing the outcome of the survey tool and its effective use as a quality-improvement instrument are long overdue.

- Combination survey methods should be tried to achieve goals where one instrument may be complementary and compensate for the weaknesses of the other instrument.

\section{Disclosure}

There are no conflicts of interest to declare.

\section{References}

1. Ruiz Moral R, Alba Dios A, Dios Guerra C, et al. Preferences, satisfaction level of patient participation in making decisions in health centre nursing clinics. Enferm Clin. 2011;21(3):136-142. Spanish.

2. Taylor K, Vandenberg S, le Huquet A, Blanchard N, Parshuram CS Parental attitudes to digital recording: a paediatric hospital survey. J Paediatr Child Health. 2011;47(6):335-339.

3. Nyandigisi A, Memusi D, Mbithi A, et al. Malaria case-management following change of policy to universal parasitological diagnosis and targeted artemisinin-based combination therapy in Kenya. PLoS One. 2011;6(9):e24781.

4. Lewis-Newby M, Curtis JR, Martin DP, Engelberg RA. Measuring family satisfaction with care and quality of dying in the intensive care unit: does patient age matter? J Palliat Med. 2011;14(12):1284-1290.

5. Kim BS, Shim DS, Lee JW, Han SH, Ko YK, Park EH. Comparison of multi-drug injection versus placebo after hallux valgus surgery. Foot Ankle Int. 2011;32(9):856-860.

6. Zhou P, Bundorf K, Le Chang J, Huang JX, Xue D. Organizational culture and its relationship with hospital performance in public hospitals in China. Health Serv Res. 2011;46(6 Pt 2):2139-2160.

7. Harrold L, Mazor K, Peterson D, Firneno C, Yood R. PS2-16: patient knowledge and beliefs concerning gout and its treatment. Clin Med Res. 2011;9(3-4):155.

8. Guadagnolo BA, Cina K, Koop D, Brunette D, Petereit DG. A pre-post survey analysis of satisfaction with health care and medical mistrust after patient navigation for American Indian cancer patients. J Health Care Poor Underserved. 2011;22(4):1331-1343. 
9. Schaupp CL, Bélanger F. A conjoint analysis of online consumer satisfaction. J Electron Commer Res. 2005;6(2):95-109.

10. Creswell JW. Qualitative Inquiry and Research Design: Choosing among Five Approaches, 2nd ed. Thousand Oaks: Sage; 2006.

11. Creswell J. Qualitative Inquiry and Research Design: Choosing among Five Traditions. Thousand Oaks: Sage; 1997.

12. Creswell JW. Research Design: Qualitative, Quantitative, and Mixed Methods Approaches, 2nd ed. Thousand Oaks: Sage; 2003.

13. Luce JM, Bindman AB, Lee PR. A brief history of health care quality assessment and improvement in the United States. West J Med. 1994;160(3):263-268.

14. Stevens R. American Medicine and the Public Interest. Berkeley: University of California Press; 1971.

15. Flexner A. Medical Education in the United States and Canada: A Report to the Carnegie Foundation for the Advancement of Teaching. Memphis: General Books; 1910.

16. Pearre AA. The history and organization of the Joint Commission on Accreditation of Hospitals. Md State Med J. 1955;4(11):698-700.

17. Roberts JS, Coale JG, Redman RR. A history of the Joint Commission on Accreditation of Hospitals. JAMA. 1987;258(7):936-940.

18. Habicht JP. Health for All by the Year 2000. Am J Public Health. 1981 May;71(5):459-461.

19. Ho S. Operations and Quality Management. London: Cengage Learning EMEA; 1999.

20. World Health Organization. Quality and Accreditation in Health Care Services: A Global Review. Geneva: WHO; 2000.

21. Goldmann D. Sustaining CQI. Int J Qual Health Care. 1997;9:1-2.

22. Ovretveit J. Total quality management in European healthcare. Int $J$ Qual Health Care. 2000;13:74-79.

23. Coulter A, Cleary P. Patients' experiences with hospital care in five countries. Health Aff (Millwood). 2001;20:24-52.

24. World Health Organization. Health Evidence Network (HEN). Copenhagen: WHO Regional Office for Europe. Available from: http://www.euro.who.int/en/what-we-do/data-and-evidence/healthevidence-network-hen/publications. Accessed January 3, 2012.

25. Shaw C. How Can Hospital Performance Be Measured and Monitored? Copenhagen: WHO Regional Office for Europe's Health Evidence Network (HEN); 2003.

26. Schauffler H, Mordavsky J. Consumer reports in health care: do they make a difference? Annu Rev Public Health. 2001;22:69-89.

27. Sitzia J. How valid and reliable are patient satisfaction data? An analysis of 195 studies. Int J Qual Health Care. 1999;11:319-328.

28. Jordan H, Carr D, Joseph C, White A. Costs and Benefits of HCAHPS: Final Report. Cambridge, MA: Abt Associates; 2005.

29. Rhode Island Department of Health Care Quality Steering Committee. Quality Hospital Care: What Does it Mean? The Results of Surveys and Focus Groups with Consumers and Health Professionals in Rhode Island. Providence: Rhode Island Department of Health; 1999.

30. Stewart M, Brown J, Donner A, et al. The Impact of patient-centered care on outcomes. J Fam Pract. 2000;49(9):796-804.

31. Dancet E, Ameye L, Sermeus W, Welkenhuysen M. The ENDOCARE questionnaire (ECQ): a valid and reliable instrument to measure the patient-centeredness of endometriosis care in Europe. Hum Reprod. 2011;26(11):2988-2999.

32. Zhu Z, Sivakumar K, Parasuraman A. A mathematical model of service failure and recovery strategies. Decision Sci. 2004;35(3):493-525.

33. Welch SJ. Twenty years of patient satisfaction research applied to the emergency department: a qualitative review. Am J Med Qual. 2010; 25(1):64-72.

34. Gallagher P. Development of a new patient-based measure of pediatric ambulatory care. Pediatrics. 2009;124(5):1348-1354.

35. Iversen MG. Do surgical patients differ in the way they prioritise aspects of hospital care? Scand J Public Health. 2009;37(3):295-303.

36. Bokhour BG. Improving methods for measuring quality of care: a patient-centered approach in chronic disease. Med Care Res Rev. 2009;66(2):147-166.
37. Schiff JH, Russ N, Ihringer K. Paediatric Perianesthesia Questionnaire: development and data from eight hospitals across Germany. Br JAnaesth. 2011;106(1):88-95.

38. Picker Institute Europe [homepage on the Internet]. Available from: http://www.pickereurope.org. Accessed September 2, 2005.

39. Hospital Consumer Assessment of Healthcare Providers and Systems. Patients' Perspectives of Care Survey. Baltimore: Centers for Medicare and Medicaid Services. Available from: https://www.cms.gov/Medicare/ Quality-Initiatives-Patient-Assessment-Instruments/HospitalQualityInits/ HospitalHCAHPS.html. Accessed April 11, 2012.

40. Elliott MN, Zaslavsky AM, Goldstein E, et al. Effects of survey mode, patient mix, and nonresponse on CAHPS hospital survey scores. Health Serv Res. 2009;44(2 Pt 1):501-518.

41. Marshall MN, Shekelle PG, Leatherman S, Brook RH. The public release of performance data. JAMA. 2000;283(14):1866-1874.

42. Hibbard J, Stockard J, Tusler M. Hospital performance reports: impact on quality, market share, and reputation. Health Aff (Millwood). 2005;24(4):1150-1160.

43. Sofaer S, Crofton C, Goldstein E, Hoy E, Crabb J. What do consumers want to know about the quality of care delivered in hospitals? Health Serv Res. 2005;40(6 Pt 2):2018-2036.

44. Hibbard J, Slovic, P, Peters E, Finucane M. Strategies for reporting health plan performance information to consumers: evidence from controlled studies. Health Serv Res. 2002;37:291-313.

45. Jenkinson C, Coulter A, Bruster S. The Picker Patient Experience Questionnaire: development and validation using data from in-patient surveys in five countries. Int J Qual Health Care. 2002;14(5):353-358.

46. Picker Institute. The Picker Institute Implementation Manual. Boston: Picker Institute; 1999.

47. Streiner D, Norman G. Health Measurement Scales. A Guide to their Development and Use, 2nd ed. Oxford: Oxford University Press; 1995.

48. Jenkinson C, McGee H. Health Status Measurement: A Brief but Critical Introduction. Oxford, UK: Radcliffe Medical Press; 1998.

49. Kumar G. Assessment of hospice patients' goals of care at the end of life. Am J Hosp Palliat Care. 2011;28(1):31-34.

50. Cleary P, Edgman-Levitan S, McMullen W, Delbanco T. The relationship between reported problems and summary evaluations of hospital care. QRB Qual Rev Bull. 1992:18(2):53-59.

51. Nunnally J, Bernstein H. Psychometric Theory, 3rd ed. New York: McGraw-Hill; 1994.

52. Gerteis M, Edgman-Levitan S. Through the Patient's Eyes: Understanding and Promoting Patient-Centered Care. New York: Jossey-Bass; 1993.

53. Blendon RJ, Buhr T, Elaine F. Disparities in physician care: experiences and perceptions of a multi-ethnic America. Health Aff (Millwood). 2008;27(2):507-517.

54. Hargraves JL, Wilson IB, Zaslavsky A, et al. Adjusting for patient characteristics when analyzing reports from patients about hospital care. Med Care. 2001;39(6):635-641.

55. Danielsen K, Bjertnaes OA, Garratt A, Forland O, Iversen HH, Hunskaar $\mathrm{S}$. The association between demographic factors, user reported experiences and user satisfaction: results from three casualty clinics in Norway. BMC Fam Pract. 2010;11:73.

56. Shi L, Macinko J. Changes in medical care experiences of racial and ethnic groups in the United States, 1996-2002. Int J Health Serv. 2008;38(4):653-670.

57. Hicks LS, Ayanian JZ, Orav EJ, et al. Is hospital service associated with racial and ethnic disparities in experiences with hospital care? Am J Med. 2005;118(5):529-535.

58. Suchman EA, Guttman L. A Solution to the problem of question "bias". Public Opin Q. 1947;11(3):444-445.

59. Creswell J. Research Design: Qualitative and Quantitative Approaches. London: Sage; 1994.

60. Barr DA. Race/ethnicity and patient satisfaction. Using the appropriate method to test for perceived differences in care. J Gen Intern Med. 2004;19(9):937-943. 
61. Young GJ, Meterko M, Desai KR. Patient satisfaction with hospital care: effects of demographic and institutional characteristics. Med Care. 2000;38(3):325-334.

62. Ly DP, Glied SA. Disparities in service quality among insured adult patients seen in physicians' offices. J Gen Intern Med. 2010;25(4):357-362.

63. Flores G, Olson L, Tomany-Korman SC. Racial and ethnic disparities in early childhood health and health care. Pediatrics. 2005;115(2): e183-e193.

64. Shone LP, Dick AW, Brach C, et al. The role of race and ethnicity in the State Children's Health Insurance Program (SCHIP) in four states: are there baseline disparities, and what do they mean for SCHIP? Pediatrics. 2003;112(6 Pt 2):e521.

65. Flores G, Tomany-Korman SC, Olson L. Does disadvantage start at home? Racial and ethnic disparities in health-related early childhood home routines and safety practices. Arch Pediatr Adolesc Med. 2005;159(2):158-165.

66. Shone LP, Dick AW, Klein JD, Zwanziger J, Szilagyi PG. Reduction in racial and ethnic disparities after enrollment in the State Children's Health Insurance Program. Pediatrics. 2005;115(6):e697-e705.

67. Edelstein BL. Disparities in oral health and access to care: findings of national surveys. Ambul Pediatr. 2002;2(Suppl 2):141-147.

68. Mayberry RM, Mili F, Ofili E. Racial and ethnic differences in access to medical care. Med Care Res Rev. 2000;57 Suppl 1:108-145.

69. Rosenheck R, Wilson NJ, Meterko M. Influence of patient and hospital factors on consumer satisfaction with inpatient mental health treatment. Psychiatr Serv. 1997;48(12):1553-1561.

70. Carlson MJ, Blustein J, Fiorentino N, Prestianni F. Socioeconomic status and dissatisfaction among HMO enrollees. Med Care. 2000; 38(5):508-516.

71. Mazor KM, Clauser BE, Field T, Yood RA, Gurwitz JH. A demonstration of the impact of response bias on the results of patient satisfaction surveys. Health Serv Res. 2002;37(5):1403-1417.

72. Etter JF, Perneger TV. Analysis of non-response bias in a mailed health survey. J Clin Epidemiol. 1997;50(10):1123-1128.

73. Puhani PA. The Heckman correction for sample selection and its critique. J Econ Surv. 2000;14(1):53-68.

74. Barkley WM, Furse DH. Changing priorities for improvement: the impact of low response rates in patient satisfaction. Jt Comm J Qual Improv. 1996;22(6):427-433.

75. Keller S, O’Malley AJ, Hays RD, et al. Methods used to streamline the CAHPS Hospital Survey. Health Serv Res. 2005;40(6 Pt 2): 2057-2077.

76. Lisa A Guion, David C Diehl, McDonald D. Triangulation: Establishing the Validity of Qualitative Studies. University of Florida; 2011. Available from: http://edis.ifas.ufl.edu/fy394. Accessed January 15, 2012.

77. Thurmond V. The point of triangulation. J Nurs Scholarsh. 2001; 33(3):254-256.

78. Macera CA, Jackson KL, Davis DR, Kronenfeld JJ, Blair SN. Patterns of non-response to a mail survey. J Clin Epidemiol. 1990;43(12): 1427-1430.

79. Gayet-Ageron A, Agoritsas T, Schiesari L, Kolly V, Perneger TV. Barriers to participation in a patient satisfaction survey: who are we missing? PLoS One. 2011;6(10):e26852.

80. O’Malley AJ, Zaslavsky AM, Elliott MN, Zaborski L, Cleary PD. Case-mix adjustment of the CAHPS Hospital Survey. Health Serv Res. 2005;40(6 Pt 2):2162-2181.

81. Gold MR, Hurley R, Lake T, Ensor T, Berenson R. A national survey of the arrangements managed-care plans make with physicians. New Engl J Med. 1995;333(25):1678-1683.

82. Klein M, Einstein A. The myth of customer satisfaction. Strategy + Business. 2003;30:1-2.

83. Bill C. Three reasons to be wary of customer satisfaction surveys. Marketing Profs. January 8, 2008. Available from: http://www.marketingprofs.com/8/reasons-wary-of-customer-satisfaction-surveys-cusick. asp. Accessed January 25, 2012.
84. Langley G, Moen R, Nolan KM. The Improvement Guide, 2nd ed. New York: Jossey-Bass; 1996.

85. Mcdonald F, Dierkes M. The importance of power in partnership relationships. J Gen Manag. 1999;25(10):43-59.

86. Boan D, Funderburk F. Healthcare Quality Improvement and Organizational Culture. Delmarva Foundation. 2003;5:1-18.

87. Maull R, Brown P, Cliffe R. Organisational culture and quality improvement. Int J Op Pr. 2001;21(3):302-326.

88. Gerowitz MB, Lemieux-Charles L, Heginbothan C, Johnson B. Top management culture and performance in Canadian, UK and US hospitals. Health Serv Manage Res. 1996;6(3):69-78.

89. Fontaine A, Vinceneux P, Traversat AF. Toward quality improvement in a French hospital: structures and culture. Int J Qual Health Care. 1997;9(3):177-181.

90. Denison D, Mishra A. Toward a theory of organizational effectiveness. Organ Sci. 1995;6(2):204-223.

91. Barney JB. Organizational culture: can it be a source of sustained competitive advantage? Acad Manage Rev. 1986;11(3):656.

92. Dean JW, Bowen DE. Management theory and total quality: improving research and practice through theory development. Acad Manage Rev. 1994;19(3):392-418.

93. Benson TE. Quality is not what you think it is. Industry Week. 1992; 241(19):22-34.

94. Frehr HU. From ISO 9000 to total quality management, a rough road. Hum Sys Manage. 1997;16(3):185-193.

95. Ellram L, Edis O. A case study of successful partnering implementation. Int J Purchas Mat Manage. 1996;32(4):11-19.

96. Gadde L, Snehota I. Making the most of supplier relationships. Ind Mark Manage. 2000;29(4):305-316.

97. Palmer R, Wilson JP. Maintaining the energy for commitment to quality. Training For Quality. 1995;3(2):9-13.

98. Klein AS, Masi RJ, Weidner CK. Organizational culture, distribution and amount of control, and perceptions of quality. Group Organ Manage. 1995;20(2):122-148.

99. Edwards P, Collinson M, Rees C. The determinants of employee responses to total quality management: six case studies. Organ Stud. 1998;19(3):449-475.

100. Bassi LJ, Ludwig J, McMurrer DP, Van Buren M. Profiting from Learning: Do Firms' Investments in Education and Training Pay Off? Alexandria, VA: American Society for Training and Development; 2000.

101. Goldstein H, Spiegelhalter D. League tables and their limitations: statistical issues in comparisons of institutional performance. JR Stat Soc. 1996;159:385-443.

102. Schneider E, Epstein A. Influence of cardiac-surgery performance reports on referral practice and access to care. New Engl J Med. 1996;335:251-256.

103. Golafshani N. Understanding reliability and validity in qualitative research. Qual Rep. 2003;8(4):597-607.

104. National Health Service. National Health Service patients' survey. 2003. Available from: http://www.doh.gov.uk. Accessed March 25, 2011.

105. Secretary of State for Health. The NHS Plan: A Plan for Investment, a Plan for Reform. London: Stationery Office; 2000.

106. National survey of NHS patients. National Patient Survey Programmebackground information. Last modified date: 26 May 2011 ed. London: Department of Health UK;2011.

107. Moumtzoglou A, Dafogianni C, Karra v, et al. Development and application of a questionnaire for assessing parent satisfaction with care. Int J Qual Health Care. 2000;12:331-337.

108. Lawthers A, Rózański BS, Nizankowski R, Ryś A. Using outpatient surveys to measure the quality of outpatient care in Krakow, Poland. Int J Qual Health Care. 1999;11:497-506.

109. Hansson L, Bjorkman T, Berglund I. What is important in psychiatric inpatient care? Quality of care from the patient's perspective. Qual Assur Health Care. 1993;5:41-47. 
110. Jenkinson C, Coulter A, Bruster S, Richards N. The coronary heart disease in-patient experience questionnaire (I-PEQ (CHD)): results from the survey of National Health Service patients. Qual Life Res. 2002;11(8):721-727.

111. Jenkinson C, Mayou R, Day A, Garratt A, Juszczak E. Evaluation of the Dartmouth COOP charts in a large-scale community survey in the United Kingdom. J Public Health Med. 2002;24(2):106-111.
112. Jenkinson C, Coulter A, Bruster S, Richards N, Chandola T. Patients' experiences and satisfaction with health care: results of a questionnaire study of specific aspects of care. Qual Saf Health Care. 2002;11(4):335-339.

Journal of Healthcare Leadership

Dovepress

\section{Publish your work in this journal}

The Journal of Healthcare Leadership is an international, peer-reviewed, open access journal focusing on leadership for the health profession. The journal is committed to the rapid publication of research focusing on but not limited to: Healthcare policy and law; Theoretical and practical aspects healthcare delivery; Interactions between healthcare and society and evidence-based practices;

Interdisciplinary decision-making; Philosophical and ethical issues; Hazard management; Research and opinion for health leadership; Leadership assessment. The manuscript management system is completely online and includes a very quick and fair peer-review system. Visit http://www.dovepress.com/ testimonials.php to read real quotes from published authors.

Submit your manuscript here: http://www.dovepress.com/journal-of-healthcare-leadership-journal 